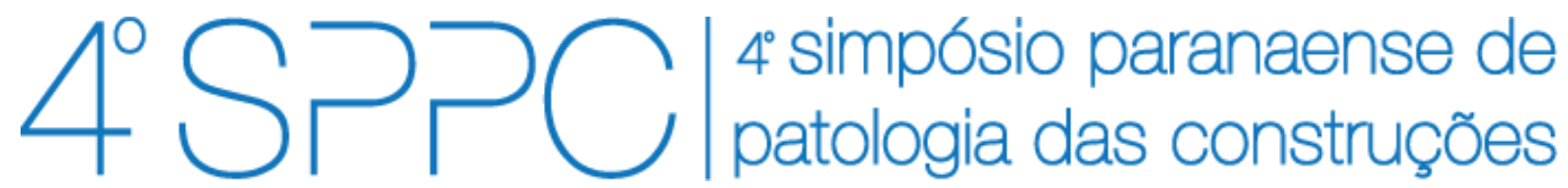

ISSN 2526-7248 artigo n. 4SPPC122, pp. 202-214, 2019

\title{
Métodos de apoio multicritério à decisão na gestão de estradas e pontes: revisão sistemática da literatura
}

\author{
Machado, Eduarda Lauck; Santos, Adriana de Paula Lacerda²; Santos Filho, Mauro \\ Lacerda $^{3}$ \\ ${ }^{1}$ Doutoranda do Programa de Pós-Graduação em Engenharia de Construção Civil, \\ Universidade Federal do Paraná (UFPR), eduarda.lauck@gmail.com \\ ${ }^{2}$ Doutora em Engenharia Civil, Professora Associada na Universidade Federal do \\ Paraná (UFPR), adrianapls@ufpr.br \\ ${ }^{3}$ Doutor em Civil Engineering, Professor Titular na Universidade Federal do Paraná \\ (UFPR), maurolacerda1982@gmail.com
}

Resumo: As atividades de intervenção em estradas e pontes, normalmente envolvem grandes investimentos que nem sempre estão disponíveis para os departamentos de transporte, o que requer desses departamentos um processo estruturado de tomada de decisão para selecionar e priorizar projetos para implementar as ações que são necessárias para manter essas infraestruturas. Assim, este artigo tem como objetivo identificar, por meio de uma revisão sistemática da literatura, quais métodos de apoio multicritérios à decisão (AMD) são utilizados na gestão de estradas e pontes. Foram identificados seis principais métodos: AHP, ANP, TOPSIS, PROMETHEE, VIKOR e SAW. O AHP foi o método mais utilizado, encontrado em 17 dos 28 artigos selecionados, apresentando-se como um método completo de ponderação de critérios e criação de ranking de preferência. Assim, um modelo de decisão para priorização de estradas e pontes para manutenção, pode ser estruturado utilizando um destes métodos AMD ou mesmo uma combinação deles, conforme as preferências do decisor.

Palavras-chave: Estrada, Ponte, Métodos de apoio multicritério à decisão.

Abstract: Roads and bridge intervention activities usually involve large investments that are not always available to transportation agencies, which requires of these agencies a complex decision-making process to select and prioritize projects to implement the actions that are necessary to maintain these infrastructures. Therefore, this paper aims to identify, with a systematic review of the literature, which multicriteria decision-making (MCDM) methods are used in the management of roads and bridges. Six main methods were identified: AHP, ANP, TOPSIS, PROMETHEE, VIKOR e SAW. AHP was the most widely used method, which was found in 17 of the 28 selected articles, presenting itself as a complete method of weighting criteria and creating preference ranking. Thus, a decision model for prioritizing roads and bridges for maintenance can be structured using one of these AMD methods or even a combination of them according to the preferences of the decision maker.

Keywords: Road, Bridge, Multicriteria decision-making methods 
MACHADO, E. L.; SANTOS, A. P. L.; SANTOS FILHO, M. L. MÉTODOS DE APOIO MULTICRITÉRIO À DECISÃO NA GESTÃO DE ESTRADAS E PONTES: REVISÃO SISTEMÁTICA DA LITERATURA. $4^{\circ}$ Simpósio Paranaense de Patologia das Construções (40 SPPC), artigo 4SPPC122, pp. 202 - 214, 2019. DOI: 10.4322/2526-7248.042

\section{Introdução}

As atividades de intervenção em estradas e pontes normalmente envolvem grandes investimentos que nem sempre estão disponíveis para os departamentos de transporte, o que requer um processo complexo de tomada de decisão para selecionar e priorizar projetos para implementar as ações que são necessárias para manter essas estruturas [1]. A tomada de decisão na gestão destas infraestruturas merece coordenação e métodos que auxiliem o decisor, de forma a atender às necessidades de todas as partes interessadas.

Os métodos de apoio multicritério à decisão (AMD) foram desenvolvidos para indicar uma preferência por uma alternativa, classificar alternativas num pequeno número de categorias e/ou classificar as alternativas numa ordem de preferência subjetiva, e resumem uma maneira de lidar com problemas complexos.

Este artigo tem como objetivo identificar quais métodos AMD são utilizados na gestão de pontes e estradas. Para tanto é utilizada uma revisão sistemática da literatura (RSL), que tem como objetivo fornecer um resumo equilibrado, claro e relevante para satisfazer uma necessidade particular de informação.

\section{Método}

Na primeira fase desta revisão definiu-se como pergunta de pesquisa: Quais métodos AMD são utilizados na gestão de estradas e pontes? Determinou-se ainda que a pesquisa será realizada através dos portais de busca Science Direct, Scopus e Periódicos Capes. Em todas as bases de dados os termos definidos para a pesquisa "multicriteria" e "decision making" foram somados (por meio do operador booleano AND) aos termos "bridge", "road" e "highway". Os termos foram buscados no título, resumos e palavras-chaves. Não se utilizou filtros por ano de publicação, área específica, nem tipo de artigo específico.

$\mathrm{Na}$ segunda fase da pesquisa, definiu-se como parâmetro principal para ser selecionado que o trabalho apresente quais métodos AMD foram utilizados em algum determinado problema de gestão de estradas ou pontes. Nesta fase, realizou-se a pré-seleção dos trabalhos, por meio da verificação de títulos e resumos para enquadramento do escopo e eliminação de redundâncias, desconsiderando-se os trabalhos duplicados. Os trabalhos pré-selecionados foram então submetidos a uma análise de conteúdo, na qual leem-se os trabalhos na íntegra e aplicam-se os critérios de seleção definidos anteriormente.

\section{Resultados e discussão}

Ao final da fase de pré-seleção dos trabalhos nos portais de buscas escolhidos, de acordo com as palavras-chave definidas, 153 trabalhos foram enquadrados dentro do escopo delimitado. Todos estes foram submetidos à fase de análise de conteúdo, e após a retirada dos trabalhos duplicados, foram selecionados 28 trabalhos que apresentaram um total de 6 métodos AMD utilizados na gestão de pontes e estradas. Na Tabela 1, são apresentados os resultados da RSL agrupados em função do método utilizado ou da combinação destes, a área de aplicação e os autores. 
MACHADO, E. L.; SANTOS, A. P. L.; SANTOS FILHO, M. L. MÉTODOS DE APOIO MULTICRITÉRIO À DECISÃO NA GESTÃO DE ESTRADAS E PONTES: REVISÃO SISTEMÁTICA DA LITERATURA. $4^{\circ}$ Simpósio Paranaense de Patologia das Construções (40 SPPC), artigo 4SPPC122, pp. 202 - 214, 2019. DOI: 10.4322/2526-7248.042

Tabela 1: Métodos AMD aplicados na gestão de infraestrutura de transportes.

\begin{tabular}{|c|c|c|c|c|c|}
\hline Método & $\begin{array}{c}\text { Área de } \\
\text { Aplicação }\end{array}$ & Autores & Método & $\begin{array}{c}\text { Área de } \\
\text { Aplicação }\end{array}$ & Autores \\
\hline \multirow{6}{*}{ AHP } & Estradas & $\begin{array}{c}{[2,3,4,5,6} \\
7,8,9,10 \mathrm{e} \\
11]\end{array}$ & SAW e AHP & Transportes & [22] \\
\hline & Ponte & {$[12,13$ e 14$]$} & $\begin{array}{c}\text { AHP } \mathrm{P} \\
\text { PROMETHEE }\end{array}$ & Infraestruturas & [23] \\
\hline & Túnel & [15 e 16] & PROMETHEE & Estradas & [24] \\
\hline & Pavimentos & [17] & $\begin{array}{c}\text { PROMETHEE e } \\
\text { VIKOR }\end{array}$ & Túnel & [25] \\
\hline & Transportes & [18] & \multirow{2}{*}{ VIKOR } & Pontes & [26] \\
\hline & Passarela & [19] & & Estradas & [27] \\
\hline ANP & Ponte & [20] & \multirow{2}{*}{ TOPSIS } & Ponte & [28] \\
\hline PROMETHEE & Ponte & [21] & & Estradas & [29] \\
\hline
\end{tabular}

\subsection{Analytic Hierarchy Process (AHP)}

O método AHP [30] consiste em um método de apoio à tomada de decisão com base em três princípios fundamentais: a decomposição da estrutura, a comparação dos julgamentos e composição hierárquica de prioridades. Os critérios, definidos por especialistas, são analisados e comparados de modo independente. Uma vez estruturada a hierarquia, as alternativas são avaliadas sistematicamente, por meio da comparação, duas a duas [30].

Uma vez que a hierarquia é construída, uma escala numérica é atribuída a cada par de $n$ alternativas $\left(A_{i}, A_{j}\right)$ pelos peritos, sendo a mais utilizada a "Escala de classificação de Saaty (1990)", em que 1 indica igual importância e 9 indica importância absoluta.

Estas escalas numéricas são utilizadas nas comparações de pares entre as alternativas no que de acordo com seu impacto sobre um elemento colocado em um nível superior da hierarquia. O termo $a_{i j k}$ representa a preferência individual do especialista $k$ em à relação alternativa $A_{i}$ em comparação com a alternativa $A_{j}$. Os julgamentos globais dos especialistas são gerados e calculados utilizando a média geométrica (Eq. 1), e são inseridos na matriz de comparação D (Eq 2):

$$
\begin{aligned}
& A_{i j}=\sqrt[n]{a_{i j 1} \times a_{i j 2} \times \ldots \times a_{i j n}} \\
& D=\left[\begin{array}{cccc}
a_{11} & a_{12} & \ldots & a_{1 n} \\
a_{21} & a_{22} & \ldots & a_{2 n} \\
\ldots & \ldots & \ldots & \ldots \\
a_{n 1} & a_{n 2} & \ldots & a_{n n}
\end{array}\right]
\end{aligned}
$$

Para ordenar as alternativas, emprega-se o resultado da aproximação da matriz de comparação $D$ usando a matriz $P$ (Eq. 3): 


$$
P=\left[\begin{array}{cccc}
p_{11} & p_{12} & \ldots & p_{1 n} \\
p_{21} & p_{22} & \ldots & p_{2 n} \\
\ldots & \ldots & \ldots & \ldots \\
p_{n 1} & p_{n 2} & \ldots & p_{n n}
\end{array}\right]
$$

O método do máximo autovetor será utilizado para determinar a análise de matrizes como (Eq. 4):

$D \cdot p=\lambda_{\max } p$

em que $\lambda_{\max }$ é o máximo autovetor de da matriz $D$.

Também é preciso determinar o índice de consistência $(\mathrm{Cl})$, que é baseado no autovetor e é calculado por meio do somatório do produto de cada elemento do autovetor pelo total da respectiva coluna da matriz comparativa original [31]. O índice de consistência por ser obtido pela Eq. 5:

$C I=\frac{\lambda_{\max }-n}{n-1}$

onde n é o número de critérios avaliados.

Para verificar se o valor encontrado do índice de consistência $(\mathrm{Cl})$ é adequado, é necessário calcular a taxa de consistência (CR), determinada pela razão entre o valor do índice de consistência $(\mathrm{Cl})$ e o índice de consistência aleatória (RC). A matriz será considerada consistente se a razão for menor que $10 \%$. Os valores de $\mathrm{RC}$, apresentados na Tabela 2, têm como base o número de critérios avaliados.

Tabela 2: $\mathrm{RC}$ ( $\mathrm{n}=$ tamanho da matriz recíproca) [30]

\begin{tabular}{ccccccccccc}
\hline $\mathbf{n}$ & $\mathbf{1}$ & $\mathbf{2}$ & $\mathbf{3}$ & $\mathbf{4}$ & $\mathbf{5}$ & $\mathbf{6}$ & $\mathbf{7}$ & $\mathbf{8}$ & $\mathbf{9}$ & $\mathbf{1 0}$ \\
\hline $\mathbf{R C}$ & 0 & 0 & 0.58 & 0,9 & 1,12 & 1,24 & 1,32 & 1,41 & 1,45 & 1,49 \\
\hline
\end{tabular}

\subsection{Analytic Network Process (ANP)}

O método ANP, que é uma generalização do AHP, considera o problema de decisão como dependente de uma rede de critérios, isto é, não exige que os critérios sejam independentes. Para tratar as dependências entre os elementos de um mesmo nível hierárquico, os problemas são então formulados em redes. A aplicação do ANP, possui três etapas, apresentadas a seguir [31].

Etapa 1. Construção das matrizes de alcance: Uma matriz de alcance é uma matriz binária em que são representadas todas as possíveis relações entre grupos ou elementos. Assim, uma rede possui duas matrizes de alcance: a matriz de alcance global (que apresenta as relações entre grupos) e a matriz de alcance local (com as relações entre os elementos). Caso o grupo i influencie o grupo j, o elemento $a_{i j} d a$ matriz de alcance global receberá o valor 1; caso não influencie, receberá o valor 0 . $\mathrm{Na}$ Fig. 1 tem-se a representação de uma matriz de alcance global e uma matriz de alcance local. 
MACHADO, E. L.; SANTOS, A. P. L.; SANTOS FILHO, M. L. MÉTODOS DE APOIO MULTICRITÉRIO À DECISÃO NA GESTÃO DE ESTRADAS E PONTES: REVISÃO SISTEMÁTICA DA LITERATURA. $4^{\circ}$ Simpósio Paranaense de Patologia das Construções (40 SPPC), artigo 4SPPC122, pp. 202 - 214, 2019. DOI: 10.4322/2526-7248.042

A matriz de alcance local é dividida em quatro blocos, sendo que cada um deles corresponde a um elemento da matriz de alcance global. Logo, se a matriz de alcance global tiver um elemento nulo, então os elementos do bloco correspondente na matriz de alcance local serão também todos nulos [33].

\begin{tabular}{|c|c|c|c|c|c|c|c|c|c|c|c|c|}
\hline \multirow[t]{8}{*}{ a) } & & $\mathrm{C} 1$ & $\mathrm{C} 2$ & C3 & $\mathrm{C} 4$ & A1 & A2 & A3 & b) & & & \\
\hline & Critério 1 & 0 & 1 & 1 & 1 & 1 & 1 & 1 & & & & \\
\hline & Critério 2 & 1 & 0 & 1 & 1 & 1 & 1 & 1 & & & Critérios & Alternativas \\
\hline & Critério 3 & 1 & 1 & 0 & 1 & 1 & 1 & 1 & & Critérios & 1 & 1 \\
\hline & Critério 4 & 1 & 1 & 1 & 0 & 1 & 1 & 1 & & Alternativas & 1 & 1 \\
\hline & Alternativa 1 & 1 & 1 & 1 & 1 & 1 & 0 & 0 & & & & \\
\hline & Alternativa 2 & 1 & 1 & 1 & 1 & 0 & 1 & 0 & & & & \\
\hline & Alternativa 3 & 1 & 1 & 1 & 1 & 0 & 0 & 1 & & & & \\
\hline
\end{tabular}

Figura 1 - a) Matriz de alcance global e b) Matriz de alcance local [31].

Etapa 2. Julgamentos: Os julgamentos são realizados aos pares entre os grupos, e depois entre os elementos, assim como no método AHP. Os julgamentos devem ser feitos sempre considerando-se o objetivo da estrutura. Todos grupos deverão ser comparados entre si sobre sua influência, importância ou afinidade, relativa a cada grupo [32].

A comparação entre elementos é efetuada quando um elemento tem uma relação de dependência de pelo menos dois elementos de um grupo. Esta comparação tem como objetivo obter o vetor de prioridade relativa destes elementos. Já a comparação entre grupos é realizada quando há relação de dependência de seus elementos com objetivo de definir o peso de cada grupo na ponderação da supermatriz [33].

Assim, as matrizes de alcance podem fornecer, previamente, o número total de julgamentos necessários para a composição dos resultados da rede. $\mathrm{Na}$ matriz de alcance global serão necessários julgamentos entre os elementos não nulos de cada coluna, enquanto na matriz de alcance local serão necessários julgamentos entre os elementos não nulos de cada coluna em cada bloco.

Etapa 3. Síntese dos Resultados. Poderão ser obtidos autovetores das matrizes das comparações simultâneas, entre os grupos e entre os elementos. Após substituir os componentes dos autovetores na matriz de alcance global, obtém-se a matriz dos pesos e substituindo na matriz de alcance local obtém-se a supermatriz [32].

$\mathrm{Na}$, sequência, ao multiplicar os pesos dos elementos em cada bloco da supermatriz pelo peso correspondente do bloco na matriz dos pesos, obtém-se a supermatriz ponderada. De tal modo, esta matriz possuirá uma matriz limite, em que suas potências convergirão para uma matriz com todas colunas iguais entre si.

Uma vez normalizada a matriz limite, para que a soma de cada coluna em cada bloco seja igual a um, obtém-se então, a matriz final que fornece as prioridades das alternativas [33]. 
MACHADO, E. L.; SANTOS, A. P. L.; SANTOS FILHO, M. L. MÉTODOS DE APOIO MULTICRITÉRIO À DECISÃO NA GESTÃO DE ESTRADAS E PONTES: REVISÃO SISTEMÁTICA DA LITERATURA. $4^{\circ}$ Simpósio Paranaense de Patologia das Construções (40 SPPC), artigo 4SPPC122, pp. $202-214$, 2019. DOI: 10.4322/2526-7248.042

\subsection{Technique for Order Preference by Similarity to Ideal Solution (TOPSIS)}

O método TOPSIS baseia-se no conceito de que a alternativa escolhida deve ter a menor distância da solução ideal-positiva e ter a maior distância possível da solução ideal negativa. As etapas deste método são descritas a seguir [34].

Etapa 1. Construção da matriz de decisão. A matriz de decisão A composta por alternativas e critérios é descrita por (Eq. 6):

$$
A=\begin{gathered}
C_{1} \\
A_{1} \\
\cdots \\
A_{m}
\end{gathered}\left(\begin{array}{ccc}
x_{11} & \cdots & C_{n 1} \\
\vdots & \ddots & \vdots \\
x_{m 1} & \cdots & x_{m n}
\end{array}\right)
$$

em que $A_{1}, A_{2}, \ldots, A_{m}$ são as alternativas disponíveis, $C_{1}, C_{2}, \ldots C_{n}$ são os critérios, $\mathrm{x}_{\mathrm{ij}}$ representa o desempenho da alternativa $A_{j}$, segundo o critério $C_{j}$.

Etapa 2. Atribuição de pesos. $O$ vetor de peso $W=\left(w_{1}, w_{2}, \ldots, w_{n}\right)$ formado pelos pesos individuais para cada critério $\mathrm{Cj}$, corresponde ao somatório (Eq. 7):

$\sum_{i=1}^{n} w_{j}=1$

Etapa 3. Normalização da matriz. A normalização da matriz pode ser feita de acordo com (Eq. 8):

$p_{i j}=\frac{x_{i j}}{\sum_{i=1}^{m} x_{i j}}$, sendo $\mathrm{i}=1, \ldots, \mathrm{m} ; \mathrm{j}=1, \ldots, \mathrm{n}$

Assim, a matriz, que representa o desempenho relativo das alternativas, pode ser descrita por (Eq. 9):

$A_{n}=\left(p_{i j}\right)_{m x n}, \operatorname{com~i~}=1, \ldots, \mathrm{m} ; \mathrm{j}=1, \ldots, \mathrm{n}$

Etapa 4. Cálculo das soluções ideais positivas $A^{+}$e das soluções ideais negativas $A^{-}$. As soluções ideais positivas $A^{+}$e das soluções ideais negativas $A^{-}$podem ser calculadas por (Eq. 10, 11, 12 e 13):

$$
\begin{aligned}
& A^{+}=\left(p_{1}^{+}, p_{2}^{+}, \ldots, p_{m}^{+}\right) \\
& A^{-}=\left(p_{1}^{-}, p_{2}^{-}, \ldots, p_{m}^{-}\right)
\end{aligned}
$$

em que

$$
\begin{aligned}
p_{j}^{+} & =\left(\max _{i} p_{i j}, j \in J_{1}\right) ; \min _{i} p_{i j}, \quad \mathrm{j} \in J_{2} \\
p_{j}^{-} & =\left(\min _{i} p_{i j}, \quad j \in J_{1}\right) ; \max _{i} p_{i j}, j \in J_{2}
\end{aligned}
$$

onde $J_{1}$ e $J_{2}$ representam os critérios de benefício e de custo, respectivamente. 
Etapa 5. Cálculo das distâncias Euclidianas. As distâncias Euclidianas entre $A_{i} \mathrm{e} \mathrm{A}^{+}$ e entre $A_{i}$ e $A^{-}$são calculadas por (Eq. 14 e 15):

$$
\begin{aligned}
& d^{+}=\sqrt{\sum_{j=1}^{n} w_{j}}\left(p_{j}^{+}-p_{i j}\right)^{2}, \text { em que } \mathrm{i}=1, \ldots, \mathrm{m} \\
& d^{-}=\sqrt{\sum_{j=1}^{n} w_{j}}\left(p_{j}^{-}-p_{i j}\right)^{2}, \text { em que } \mathrm{i}=1, \ldots, \mathrm{m}
\end{aligned}
$$

Etapa 6. Cálculo da proximidade relativa. A proximidade relativa $\xi i$ para cada alternativa $A_{i}$ em relação à solução ideal positiva $A^{+}$é dada por (Eq. 16):

$\xi_{i}=\frac{d_{i}^{-}}{d_{i}^{+}+d_{i}^{-}}$

Por fim, faz-se a ordenação das alternativas.

\subsection{Preference Ranking Organization METHod of Enrichment Evaluation (PROMETHEE)}

O método PROMETHEE é um método de preferência que fornece ao decisor uma estrutura preferencial entre alternativas discretas. A variação PROMETHEE II apresenta um ranking completo de alternativas

Etapa 1. Construção da matriz de decisão: Sendo $A$ o conjunto de alternativas, para cada alternativa $a_{i} \in A, \mathrm{i}=1, \ldots, \mathrm{n}, f_{j}(a)$ é uma avaliação dessa alternativa segundo o critério $j, \mathrm{j}=1, \ldots, \mathrm{k}$. $\mathrm{O}$ conjunto dessas avaliações podem ser representadas na matriz M (Eq. 17):

$\mathrm{M}=\left[\begin{array}{cccc}f_{1}\left(a_{1}\right) & f_{2}\left(a_{1}\right) & \cdots & f_{k}\left(a_{1}\right) \\ f_{1}\left(a_{2}\right) & f_{2}\left(a_{2}\right) & \cdots & f_{k}\left(a_{2}\right) \\ \vdots & \vdots & & \\ f_{1}\left(a_{n}\right) & f_{2}\left(a_{n}\right) & \cdots & f_{k}\left(a_{n}\right)\end{array}\right]$

Etapa 2. Especificação, para cada critério $f_{j}$, de uma função de preferência generalizada $\left(P_{j}\right)$, tal que (Eq. 18):

$P_{j}: A \times A \rightarrow[0,1]$

A função de preferência de um critério descreve a forma como a preferência do decisor varia com a diferença entre os níveis de desempenho de duas alternativas em relação a esse critério. Numa comparação entre duas alternativas quaisquer, $a_{r}$ e as, pertencentes ao conjunto A, tem-se (Eq. 19):

$P_{j}\left(a_{r}, a_{s}\right)=P(x)=P\left[f\left(a_{r}\right)-f\left(a_{s}\right)\right]$

que representa a intensidade com que a alternativa $a_{r}$ é preferível a $a_{s}$, segundo o critério j. 
Quando o critério precisa ser maximizado, utiliza-se $x=f\left(a_{r}\right)-f\left(a_{s}\right)$ para definir a função de preferência. Para a minimização do critério, a função de preferência será $x$ $=f\left(a_{s}\right)-f\left(a_{r}\right)$. A intensidade de preferência deve ser calculada para cada par de alternativas, considerando todos os critérios. As funções de preferência podem ser consultadas em [35].

Etapa 3. Definição dos pesos $a_{j}(j=1,2, \ldots, n)$, que são as medidas de importância de cada critério.

Etapa 4. Calcular o índice de preferência ponderada global, $\pi\left(a_{r}, a_{s}\right)$, para todos os pares de alternativas. Este índice indica o percentual de preferência da alternativa $a r$ em relação à $a_{s}$, considerando os pesos atribuídos a casa critério, Eq. 20, 21 e 22:

$\pi\left(a_{r}, a_{s}\right)=\sum_{j=1}^{n} \alpha_{j} P_{j}\left(a_{r}, a_{s}\right)$

em que,

$\sum_{j=1}^{n} \alpha_{j}=1, \mathrm{e}$

$0 \leq \pi\left(a_{r}, a_{s}\right) \leq 1 \forall a_{r}, a_{s} \in A$

Etapa 5. Cálculo do fluxo de importância positivo, que representa a média de preferência de qualquer alternativa ar sobre as demais alternativas do conjunto $A$ (Eq. 23 e 24):

$\emptyset^{+}\left(a_{r}\right)=\frac{1}{n-1} \cdot \sum_{x \in A}^{n} \pi\left(a_{r}, x\right)$

onde,

$\emptyset^{+}: A \rightarrow[0,1]$

quanto maior $\emptyset^{+}\left(a_{r}\right)$, melhor a alternativa.

Etapa 6. Cálculo do fluxo de importância negativo, que representa a média de preferência de todas as alternativas sobre a alternativa $a_{r}$ (Eq. 25 e 26)

$\emptyset^{-}\left(a_{r}\right)=\frac{1}{n-1} \cdot \sum_{x \in A}^{n} \pi\left(x, a_{r}\right)$

onde,

$\emptyset^{-}: A \rightarrow[0,1]$

quanto menor $\emptyset^{+}\left(a_{r}\right)$, melhor a alternativa.

O ranking é obtido calculando-se o fluxo líquido, que é a diferença entre o fluxo positivo e negativo, para cada alternativa. 
MACHADO, E. L.; SANTOS, A. P. L.; SANTOS FILHO, M. L. MÉTODOS DE APOIO MULTICRITÉRIO À DECISÃO NA GESTÃO DE ESTRADAS E PONTES: REVISÃO SISTEMÁTICA DA LITERATURA. $4^{\circ}$ Simpósio Paranaense de Patologia das Construções (40 SPPC), artigo 4SPPC122, pp. 202 - 214, 2019. DOI: 10.4322/2526-7248.042

\subsection{Vlšekriterijumsko KOmpromisno Rangiranje (VIKOR)}

O Método VIKOR [35] se resume em elaborar um ranking a partir de um conjunto de alternativas de critérios contraditórios presentes, apresentando assim uma classificação com base na medida de proximidade com a solução ideal. O ranking de compromisso, obtido através do VIKOR possui quatro etapas [36].

Etapa 1. Determinar os maiores valores $f_{i}^{+}$e os menores valores $f_{i}^{-}$de toda a função (Eq. 27 e 28):

$f_{i}^{+}=\max f_{i j}(i=1,2, \ldots, n)$

$f_{i}^{-}=\operatorname{minf}_{i j}(i=1,2, \ldots, n)$

onde,

$f_{i}^{+}$: maior valor apresentado pelo indicador $\mathrm{i}$;

$f_{i}$ : menor valor apresentado pelo indicador $\mathrm{i}$.

Etapa 2. Calcular os valores $S_{j}$ (grupo de utilidade máxima) e $R_{j}$ (peso individual mínimo), onde $j=1,2, \ldots, J$, nas relações, em que wisão os pesos dos critérios (Eq. 29 e 30).

$$
\begin{aligned}
& S_{i} \sum_{j=1}^{m}\left[w_{j} \frac{f_{j}^{+}-f_{i j}}{f_{j}^{+}-f_{j}^{-}}\right] \\
& R_{i}=\max _{j}\left[w_{j} \frac{f_{j}^{+}-f_{i j}}{f_{j}^{+}-f_{j}^{-}}\right]
\end{aligned}
$$

onde:

$S_{j}$ : grupo de utilidade máxima;

$\mathrm{w}_{i}$ : peso estratégico (normalmente $\mathrm{v}=0.5$ );

$f_{i j}$ : o valor do indicador $\mathrm{i}$; e

$R_{j}$ : peso individual mínimo.

Etapa 3. Calcular os valores $Q_{j}$, onde $j=1,2, \ldots, \mathrm{J}$, pela relação, em que $S^{+}=$min $S_{j}$, $S=\max S_{j}, R^{+}=\min R_{j}, R^{-}=\max R_{j}$, o $v$ é introduzido como peso de estratégia, geralmente utilizado como $v=0,5$ (Eq. 31).

$Q_{J}=\frac{v\left(S_{j}-S^{+}\right)}{\left(S^{-}-S^{+}\right)}+\frac{(1-v)\left(R_{j}-R^{+}\right)}{\left(R^{-}-R^{+}\right)}$

onde:

$v=0,5$

$Q_{j}$ : score final;

$S^{+}:$menor grupo de utilidade máxima;

$S:$ maior grupo de utilidade máxima;

$R^{+}$: menor peso individual mínimo; e

$R$ : maior peso individual mínimo. 
MACHADO, E. L.; SANTOS, A. P. L.; SANTOS FILHO, M. L. MÉTODOS DE APOIO MULTICRITÉRIO À DECISÃO NA GESTÃO DE ESTRADAS E PONTES: REVISÃO SISTEMÁTICA DA LITERATURA. $4^{\circ}$ Simpósio Paranaense de Patologia das Construções (40 SPPC), artigo 4SPPC122, pp. 202 - 214, 2019. DOI: 10.4322/2526-7248.042

Etapa 4. Classificar as alternativas de forma decrescente, pelos valores obtidos por $S, R$ e $Q$. Como resultado obtêm-se três listas de classificação. Porem podem ser considerados apenas os valores obtidos por $Q$.

\subsection{Simple Additive Weighting (SAW)}

O método SAW, agrega de maneira aditiva a contribuição dos atributos, ao multiplicar o valor do atributo por seu peso e somar posteriormente os produtos obtidos para cada alternativa. Na sequência, atribui-se a cada uma das alternativas um indicador equivalente à medida ponderada de todos os critérios [37].

\subsection{Uso dos métodos AMD}

O AHP foi o método mais utilizado na gestão de estradas e pontes, encontrado em 17 dos 28 artigos selecionados, apresentando-se como um método completo de ponderação de critérios e criação de ranking de preferência das alterativas, enquanto os demais permitem apenas a obtenção do ranking de preferência.

Em uma situação em que se tem muitas estradas e pontes, por exemplo, pode-se realizar primeiramente uma categorização destas segundo sua criticidade, em seguida, utilizar o método AHP para ponderar os critérios considerados importantes na priorização da intervenção, e por fim um dos demais métodos (TOPSIS, PROMETHEE, VIKOR ou SAW) para criar o ranking de preferência.

\section{Considerações finais}

O principal objetivo deste estudo foi identificar, por meio de uma revisão sistemática da literatura, quais métodos de apoio multicritério à decisão são utilizados na gestão de pontes e estradas.

Nos 28 artigos selecionados, foram identificados seis principais métodos (AHP, ANP, TOPSIS, PROMETHEE, VIKOR e SAW) utilizados principalmente para gestão de pontes, estradas, túneis e pavimentos.

Conclui-se então que, modelos de decisão para priorização de estradas e pontes para manutenção podem ser estruturados utilizando uma combinação de métodos AMD de ponderação de critérios e de criação de ranking de prioridade, conforme as preferências do decisor.

\section{Agradecimentos}

Esta pesquisa foi apoiada pelo Programa de Pós-Graduação em Engenharia e Construção civil (PPGECC) na Universidade Federal do Paraná (UFPR). 
MACHADO, E. L.; SANTOS, A. P. L.; SANTOS FILHO, M. L. MÉTODOS DE APOIO MULTICRITÉRIO À DECISÃO NA GESTÃO DE ESTRADAS E PONTES: REVISÃO SISTEMÁTICA DA LITERATURA. $4^{\circ}$ Simpósio Paranaense de Patologia das Construções (40 SPPC), artigo 4SPPC122, pp. 202 - 214, 2019. DOI: 10.4322/2526-7248.042

\section{Referências}

[1] Abu Dabous, S.; Alkass, S. (2010) A multi-attribute ranking method for bridge management. Engineering, Construction and Architectural Management, 17:3, 282-291.

[2] Klungboonkrong, P.; Taylor, M. A. P. (1999) An integrated planning tool for evaluating road environmental impacts. Computer-Aided Civil and Infrastructure Engineering, 14:5, 335-345.

[3] Kalamaras, G. S. et al. (2000) Application of multicriteria analysis to select the best highway alignment. Tunnelling and Underground Space Technology, 15:4, 415-420.

[4] Li, Z.; Sinha, K. C. (2009) Methodology for the determination of relative weights of highway asset management system goals and of performance measures. Journal of Infrastructure Systems, 15:2, 95-105.

[5] Khademi, N.; Sheikholeslami, A. (2009) Multicriteria group decision-making technique for a low-class road maintenance program. Journal of Infrastructure Systems, 16:3, 188-198.

[6] Haghighat, F. (2011) Application of a multi-criteria approach to road safety evaluation in the Bushehr province, Iran. PROMET-Traffic\&Transportation, 23:5, 341-352.

[7] Wiethoff, M. et al. (2012) A methodology for improving road safety by novel infrastructural and invehicle technology combinations. European transport research review, 4:2, 67-77.

[8] Yu, J.; Liu, Y. (2012) Prioritizing highway safety improvement projects: A multicriteria model and case study with SafetyAnalyst. Safety science, 50:4, 1085-1092

[9] Odoki, J. B. et al. (2015) A multi-criteria methodology for optimising road investments. In: Proceedings of the Institution of Civil Engineers-Transport. Thomas Telford Ltd, 34-47.

[10] Ouma, Y. O. et al. (2014) Optimization of urban highway bypass horizontal alignment: a methodological overview of intelligent spatial MCDA approach using fuzzy AHP and GIS. Advances in Civil Engineering, 2014.

[11] Sordyl, J. (2015) Application of the AHP method to analyze the significance of the factors affecting road traffic safety. Transport Problems: an International Scientific Journal, 10:2, 57-68.

[12] Abu Dabous, S.; Alkass, S. (2008) Decision support method for multi-criteria selection of bridge rehabilitation strategy. Construction Management and Economics, 26:8, 883-893, 2008.

[13] Jakiel, P.; Fabianowski, D. (2015) FAHP model used for assessment of highway $\mathrm{RC}$ bridge structural and technological arrangements. Expert Systems with Applications, 42:8, 4054-4061.

[14] Pan, N. F. (2008) Fuzzy AHP approach for selecting the suitable bridge construction method. Automation in construction, 17:8, 958-965. 
MACHADO, E. L.; SANTOS, A. P. L.; SANTOS FILHO, M. L. MÉTODOS DE APOIO MULTICRITÉRIO À DECISÃO NA GESTÃO DE ESTRADAS E PONTES: REVISÃO SISTEMÁTICA DA LITERATURA. $4^{\circ}$ Simpósio Paranaense de Patologia das Construções (40 SPPC), artigo 4SPPC122, pp. 202 - 214, 2019. DOI: 10.4322/2526-7248.042

[15] Panou, K. D.; Sofianos, A. I. (2002) A fuzzy multicriteria evaluation system for the assessment of tunnels vis-à-vis surface roads: theoretical aspects - part I. Tunnelling and underground space technology, 17:2, 195-207.

[16] Panou, K. D.; Sofianos, A. I. (2002) A fuzzy multicriteria evaluation system for the assessment of tunnels vis-à-vis surface roads: The WPMA case - part II. Tunnelling and underground space technology, 17:2, 209-219.

[17] Cafiso, S. et al. (2002) Multicriteria analysis method for pavement maintenance management. Transportation Research Record: Journal of the Transportation Research Board, 1816, 73-84.

[18] Leviäkangas, P.; Lähesmaa, J. (2002) Profitability evaluation of intelligent transport system investments. Journal of Transportation Engineering, 128:3, 276-286.

[19] Šimunović, L. et al. (2010) Selecting optimal pedestrian crossing using multicriteria decision-making. PROMET-Traffic\&Transportation, 22:2, 105-116.

[20] Chen, Z. et al. (2013) ANP experiment for demolition plan evaluation. Journal of Construction Engineering and Management, 140:2, 06013005.

[21] Balali, V. et al. (2014) Selection of appropriate material, construction technique, and structural system of bridges by use of multicriteria decision-making method. Transportation Research Record: Journal of the Transportation Research Board, 2431, 79-87.

[22] Jajac, N.; et al. (2015) Decision support for management of urban transport projects. Građevinar, 67:2, 131-141.

[23] Gervásio, H.; Silva, L. S. (2012) A probabilistic decision-making approach for the sustainable assessment of infrastructures. Expert Systems with Applications, 39:8, 7121-7131.

[24] Albuquerque, F. S.; Núñez, W. P. (2010) Critérios para a tomada de decisão em obras rodoviárias sustentáveis. Ambiente Construído, 10:3, 151-163.

[25] Vučijak, B.; et al. (2015) A. Use of multi-criteria decision aid methods for selection of the best alternative for the highway tunnel doors. Procedia Engineering,100, 656-665.

[26] Ćosić, M. et al. (2016) Multidisciplinary Approach to the Assessment of Seismic Performances and Rehabilitation of Bridges: Nonlinear Analyses, Probability Theory and Optimization Theory. Procedia Engineering, 156, 83-90.

[27] San Cristóbal, J. R. (2011) Contractor selection using multicriteria decisionmaking methods. Journal of Construction Engineering and Management, 138:6, 751-758.

[28] Markiz, N.; Jrade, A. (2014) Integrating a fuzzy-logic decision support system with bridge information modelling and cost estimation at conceptual design stage of concrete box-girder bridges. International Journal of Sustainable Built Environment, 3:1, 135-152.

[29] Bao, Q. et al. (2012) Improved hierarchical fuzzy TOPSIS for road safety performance evaluation. Knowledge-based systems, 32, 84-90. 
[30] Saaty, T. L. (1990) How to make a decision: the analytic hierarchy process. European journal of operational research, 48:1, 9-26.

[31] Vargas, R. V. (2010) Utilizando a programação multicritério (Analytic Hierarchy Process-AHP) para selecionar e priorizar projetos na gestão de portfólio. In: PMI Global Congress. p. 31.

[32] Salomon, V.; Montevecchi, J. (1998) Método de análise em redes: sucessor do método de análise hierárquica. Produto \& Produção, 3:2, 107-117.

[33] Rangel, D. A. (2012) Proposta de um procedimento para identificar, avaliar e priorizar riscos em cadeias de suprimentos. Dissertação (mestrado), Universidade Federal da Paraíba, João Pessoa, Brasil.

[34] Krohling, R. A.; Souza, T. (2011) Dois exemplos da aplicação da técnica TOPSIS para tomada de decisão. Revista de Sistemas de Informação da FSMA, 1:8, 31-35.

[35] Brans, J. P.; et al. (1986) How to select and how to rank projects: The PROMETHEE method. European journal of operational research, 24:2, 228-238.

[36] Opricovic, S. (2011) Fuzzy VIKOR with an application to water resources planning. Expert Systems with Applications, 38:10, 12983-12990.

[37] Medina, J. (2007) Impacto económico del regadío sobre el desarrollo local en España. In: Reunión Anual Asepelt España, 21. Valladolid. 10, 1003 - 115. 